\title{
Gold Catalyzed Growth of Three-dimensional Hierarchical ZnO Nanobelts
}

\author{
Hongyang Liu*, Dinghao Tang**** and Jingyue (Jimmy) Liu*** \\ * Center for Nanoscience, ** Department of Physics \& Astronomy, University of Missouri-St. \\ Louis, One University Boulevard, St. Louis, Missouri 63121, USA (liuj@umsl.edu)
}

The wide band-gap (3.37 eV), semiconducting $\mathrm{ZnO}$ has many important technological applications in photonics, optoelectronics, photovoltaics, energy storage, sensing and catalysis [1]. Although various types of $\mathrm{ZnO}$ nanostructures have been synthesized [2], it is still a challenge to reliably synthesize large amount of $\mathrm{ZnO}$ nanostructures with high-surface area and controlled surface properties. In order to utilize the unique properties of $\mathrm{ZnO}$ nanostructures for practical applications, especially for applications in catalysis, our group has been developing synthesis protocols that can generate large quantities of $\mathrm{ZnO}$ nanostructures with defined surface properties for applications in energy, sensing and drug delivery. Here, we report a new synthesis route to grow high-density and high-surface area 3D hierarchical $\mathrm{ZnO}$ nanobelts.

The 3D hierarchical $\mathrm{ZnO}$ nanobelts were synthesized in a high temperature tube furnace. The experimental setup was similar to the synthesis of $\mathrm{ZnO}$ nanobelts as reported in literature [3]. We used the pre-synthesized $\mathrm{ZnO}$ tetrapods, coated with $\mathrm{Au}$ nanoparticles, as the seed to grow $\mathrm{ZnO}$ nanobelts. Fig. 1 is a low magnification SEM image of the $\mathrm{ZnO}$ tetrapods dispersed onto the alumina substrate. The whole assemble was put into the tube furnace to collect $\mathrm{ZnO}$ nanostructures. We expected to grow $\mathrm{ZnO}$ nanobelts on the surfaces of the $\mathrm{ZnO}$ tetrapods.

Figure 2a shows a low magnification SEM image of the synthesized material. It can be clearly seen that all the tetrapod seed crystals were covered with various sizes of $\mathrm{ZnO}$ nanobelts after the synthesis process. Figure $2 \mathrm{~b}$ shows an individual tetrapod decorated with $\mathrm{ZnO}$ nanobelts on all of its four legs. The sizes of the $\mathrm{ZnO}$ nanobelts vary significantly, ranging from 0.5 to 5 micrometers in width and 2 to 20 micrometers in length; the average thickness of the $\mathrm{ZnO}$ nanobelts is about $25 \mathrm{~nm}$. The growth direction of most of the $\mathrm{ZnO}$ nanobelts is perpendicular to the surfaces of the $\mathrm{ZnO}$ tetrapod as shown clearly in Fig. 2c. High resolution backscattered electron images and TEM images revealed that small $\mathrm{Au}$ nanoparticles were located at the tips of all the $\mathrm{ZnO}$ nanobelts, suggesting that the Au nanoparticles acted as a catalyst to facilitate the secondary growth of the 3D hierarchical $\mathrm{ZnO}$ nanobelts on the surfaces of the original $\mathrm{ZnO}$ tetrapod crystals. Figure 3 shows schematic diagrams illustrating the $\mathrm{Au}$ catalyzed growth processes of the $\mathrm{ZnO}$ nanobelts on the original $\mathrm{ZnO}$ tetrapods. Detailed growth processes and the morphology dependence on the experimental parameters will be discussed [4].

\section{References}

[1] J. Jagadish, S.J. Pearton (Eds.), Zinc Oxide Bulk, Thin Film and Nanostructures, Elsevier, 2006.

[2] Z.L. Wang, J. Phys.: Condens. Matter 16 (2004) R829.

[3] Z. W. Pan et al., Science 291 (2001) 1947.

[4] This research was supported by the University of Missouri-St. Louis. 

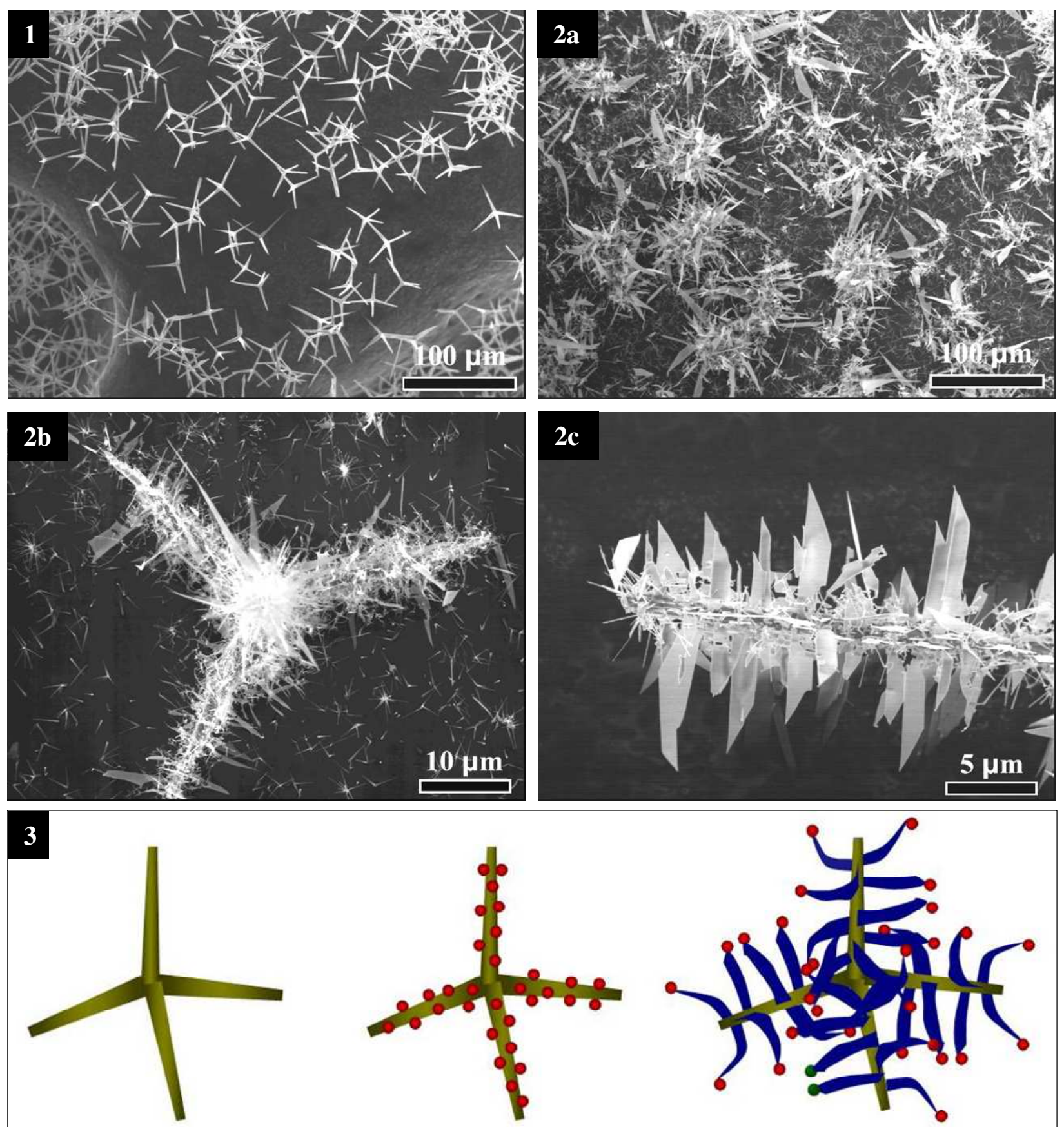

Fig.1. Low magnification SEM image shows the $\mathrm{ZnO}$ tetrapods coated with Au nanoparticles.

Fig.2. Low magnification SEM image (a) shows the growth of 3D hierarchical $\mathrm{ZnO}$ nanobelts grown on the surfaces of $\mathrm{ZnO}$ tetrapods, (b) an individual $\mathrm{ZnO}$ tetrapod decorated with $\mathrm{ZnO}$ nanobelts of various sizes and (c) an individual leg of a tetrapod showing that the growth direction of the $\mathrm{ZnO}$ nanobelts is perpendicular to the surface of the $\mathrm{ZnO}$ tetrapod.

Fig.3. Schematic diagrams illustrate the original tetrapod (left panel), tetrapod coated with Au (middle panel) and the Au catalyzed growth of 3D hierarchical $\mathrm{ZnO}$ nanobelts (right panel). 